\title{
A consultant-led group for in-patients in a medium secure unit
}

\section{AIMS AND METHOD}

The first 14 months of a consultantled weekly group for in-patients in a medium secure unit are described.

\section{RESULTS}

The group's (voluntary) attendance averaged more than $60 \%$ of the team's patients over 50 sessions. The weekly attendance increased significantly in the second seven-month period compared with the first (mean numbers of patients (s.d.) 10.4 (0.6) v. $8.1(0.8))$. An eclectic approach, with supportive, educational and interpretive elements, evolved as most suitable for the heterogeneous group of patients involved.

\author{
CLINICAL IMPLICATIONS \\ The group represented a safe, \\ efficient and practical way of \\ responding to patients' wishes for \\ consultant contact, information, \\ support and also of observing the \\ individual's mental state and ability \\ to interact in a complex social \\ setting.
}

Despite the widespread acceptance of the multidisciplinary approach to the care of psychiatric patients, user groups frequently complain of insufficient direct contact between patient and consultant. From the latter's perspective, direct observation of and interaction with his or her patients is indispensable, but may not be best effected by the patient's brief attendance at weekly team meetings nor by irregular interviews of varying duration.

\section{Method}

We decided to test the feasibility of a weekly in-patient group, run by the consultant and two other members of the multi-disciplinary team, initially a senior nurse and an occupational therapist. Three is an unconventional number of therapists but more disciplines were thereby represented and the need for a lone therapist to lead the group because of leave was obviated. Over 14 months, a nursing assistant, a music therapist and a social worker also took on the role of group therapist.

The group was open to all in-patients of one multidisciplinary team (14-16 in-patients) from all the five unit wards, ranging in function from intensive care to rehabilitation. The patients were encouraged to attend unless there was a compelling reason not to do so. The group took place on Monday afternoons, 3.30-4.15 pm off the wards. Patients were reminded 10-20 minutes beforehand. They were accompanied to the group room when necessary. Each session was written up immediately afterwards for the purpose of monthly supervision by an experienced group therapist.

The patients were given a written statement indicating time, venue, names of the therapists, aims and rules and an outline of how the group would function. The outline stated: "the group will meet weekly in the Therapy Centre for 45 minutes. The group will start and finish promptly. All patients under Dr Lucas's care are encouraged to attend and to stay for the duration of the group". The following aims were identified: (a) a chance to meet and discuss your progress with members of the team responsible for your care; (b) to give and receive support; (c) to express thoughts and feelings about your present situation; (d) to discuss the implications of living in a secure unit.

The written rules were: (a) you will be expected to attend the group punctually and stay for the duration of the 45-minute session; (b) the group will be given advance notice of holiday breaks; (c) you are expected to respect the confidential nature of personal issues raised in the group; and (d) the expression of strong feelings will be verbal only. Threatening others, damaging furniture or similar behaviour is unacceptable.

\section{Characteristics of patients}

Seventeen of the patients were male, two female. Fourteen patients suffered from paranoid schizophrenia, one from simple schizophrenia, two from delusional disorder and two from affective psychosis. The majority of patients additionally experienced significant difficulties in the personality domain usually manifested in impulsive antisocial behaviour, substance misuse and in the violence of their offences. Nine patients were subject to hospital orders with restrictions (Section 37/41); two were sentenced prisoners, transferred under Section 47/49; there was one remand prisoner, transferred under Section 48/49, three were subject to hospital orders without restrictions (Section 37); four were detained under Section 3 of the Mental Health Act 1983.

\section{Results}

\section{Attendance}

The data pertain to 50 sessions between May 1998 and July 1999. Nineteen patients attended the group with a range of attendance from 3-45 sessions (mean per patient (s.d.) 24.2 (4.6)). No patient refused ever to attend. Five therapists were involved with the group and only the consultant was consistently present throughout the period described. Average monthly attendances for 1998 were as follows: May, 8.5; June, 9.2; July, 8.0; (August: break) September, 8.8; October, 7.8; November, 7.6; December, 7.0. For 1999: January, 9.5; February, 
10.5; March, 11.3; April, 11.0; May, 10.3; June, 10.0; July, 10.3. When the seven months of 1999 were compared with the seven months of 1998 there was a statistically special articles significant increase (mean number of patients attending (s.d.) 10.4 (0.6) v. 8.1 (0.8) $P<0.01$, Mann-Whitney Utest).

\section{Security}

It was never necessary to call for emergency support although personal alarms were worn. When patients left prematurely (three occasions, two patients) they were accompanied by a therapist back to their ward. No episode of self-harm or harm to another occurred during the group or the subsequent two hours.

\section{Group process}

The agenda for each group was determined by the patients. They raised issues concerning the individual, their families, the unit, the community and also wider social and political matters. Many used the group as an opportunity for direct questioning of the consultant about their treatment, for making requests and for ensuring that information reached the consultant. Responding to questions focused on individuals, the consultant could provide educational information. In declining to make decisions, he could emphasise the multi-disciplinary nature of decision-making.

\section{Discussion}

The differences between our patients and those traditionally believed to benefit from group approaches (Yalom, 1985) together with the view that forensic patients do not readily develop cohesive or otherwise therapeutic group dynamics (Stein \& Brown, 1991) may explain the fact that a consultant-led group for mediumsecure in-patients has not previously been reported. It is the author's impression that consultant-led groups are currently uncommon in UK medium secure units. The present report indicates the feasibility of such a group. No attempt has been made to assess the therapeutic effect of the group nor to ascertain the patients' views, both of which would have required a different design. The patients were encouraged but not pressured into attending and some patients attended only very occasionally. A mean attendance of more than $60 \%$ is suggestive of the importance of the group to the patients. The improvement in attendance in the second seven-month period suggested the group had become more established and possibly more valued.

Yalom (1985) compared an 'acute in-patient therapy group' with 'conventional out-patient group psychotherapy'. The former was shorter term, had a higher turnover of staff and patients who were often more disturbed, frequently reluctant and heterogeneous. Yalom suggested that therapeutic technique should be more active, supportive, structured and oriented to the 'here-and-now'. The group described in the present paper occupies a position intermediate between the traditional out-patient psychotherapy group and Yalom's acute inpatient group. As in the former, its members are available for periods of above 18 months and the majority are not (no longer) acutely psychotic. Like the latter, its members are often involuntary in-patients and lack insight. In terms of therapists, the consultant was a constant presence while his co-therapists changed. In contrast to Yalom's suggestions for an 'acute' group, we decided not to impose an agenda, nor obvious structure, in part because of the shorter duration of each session ( 45 minutes $v .75$ minutes).

Cox (1996), discussing his psychotherapeutic approach to patients in maximum security, emphasised the 'continuum between interpretive and supportive psychotherapy' as well as the 'ubiquity of transference and countertransference phenomena'. Our intention was that our group should be supportive and educational (Bloch, 1986) but we anticipated that strong negative emotions, especially those towards the consultant or the unit, would require 'attuned empathic holding' (Cox, 1996). In several sessions, the consultant was the subject of angry (verbal) attacks but, on almost all such occasions, a tense atmosphere gave way to one of less confrontation after the consultant's response. Our experience appeared similar to that of Elliott (1997) who pointed out how the 'inevitable intense, negative feelings . . . experienced towards the therapist' are made more bearable if they are survived in the context of the more neutral or positive feelings available within a group. This allows the group members 'to slowly integrate diverse parts of themselves and others' (Elliott, 1997). The fact that the consultant was seen to be capable of withstanding such intense attacks without retaliating or collapsing appeared beneficial.

Experience suggests that most patients view the consultant as vested with power and authority. In the context of a medium secure unit, Persaud \& Meux (1994) demonstrated the potential containing effect of such authority by measuring the increased disruption during the consultant psychiatrist's temporary absence. Many patients in medium security complain that they have insufficient contact with the consultant. While this may have a basis in reality, it may also reflect what Kirtchuk \& Haworth (1996) have designated the "very distorted concepts of authority" in forensic psychiatry patients. Our consultant-led group provided a regular weekly session of fixed duration and format in which the consultant was consistently available. It seems reasonable to hypothesise that such 'reality', particularly the availability of the consultant, might influence the projective phenomena underlying these 'distorted concepts of authority'.

While generally beneficial, the consultant's presence may have led to a focus on concrete issues and thereby affected the group's development as a space for imaginative reflection. A longer duration may be required for such more 'playful' communications to emerge in a group of patients such as ours (Van Velsen, 1999, personal communication).

It appeared that the consultant, the multidisciplinary team and the patients benefited from the 
weekly consultant-led group. From the point of view of the consultant and the multi-disciplinary team, good quality information as to the individual patient's mental state was obtained each week. In addition, observation of the patient's social interaction in the group enabled a further dimension of functioning to be assessed. From the patients' point of view, the group represented a safe, efficient and practical way of responding to their wishes for consultant contact and of meeting some of their needs for information, support and 'talking treatments' (Wood, 1993).

\section{Acknowledgements}

I thank my co-therapists Annette Wynne, Geoff Haines, Nasima Haque, Claire Hobbes, Sophie Mayes and Danny Lawlor for their efforts and support, Cleo Van Velsen for her very helpful suggestions and encouragement and Hilary Scurlock for advice on an earlier draft of the paper.

\section{References}

BLOCH, S. (1986) Supportive psychotherapy. In An Introduction to the Psychotherapies (ed S. Bloch), pp. 252-277. Oxford: Oxford University Press.

COX, M. (1996) Supportive and interpretive psychotherapy in diverse contexts. In Forensic Psychotherapy Vol. II (eds C. Cordess \& M. Cox), pp. 83-93. London: Jessica Kingsley.

ELLIOTT, B. (1997) The treatment of alcohol dependency in groups. In $A$ Practical Guide to Forensic Psychotherapy (eds E. Welldon \& C. Van Velsen) pp. 130-135. London: Jessica Kingsley.

KIRTCHUK, G. \& HAWORTH, H. (1996) Psychodynamics and the regional

secure unit. In Forensic Psychotherapy Vol. II (eds C Cordess \& M. Cox) pp. 423-431. London: Jessica Kingsley. PERSAUD, R. D. \& MEUX, C. J. (1994) The psychopathology of authority and its loss: the effect on a ward of losing a consultant psychiatrist. British Journal of Medical Psychology, 67,1-11.

STEIN, E. \& BROWN, J. D. (1991) Group therapy in a forensic setting. Canadian Journal of Psychiatry, 36, 718-722.

WOOD, D. (1993) The Power of Words: Uses and Abuses of Talking Treatments. London: Mind.

YALOM, I. (1985) TheTheory and Practice of Group Psychotherapy. New York: Basic Books.

Philip Lucas Consultant in Forensic Psychiatry, John Howard Centre, 2 Crozier Terrace, London E9 6AT 